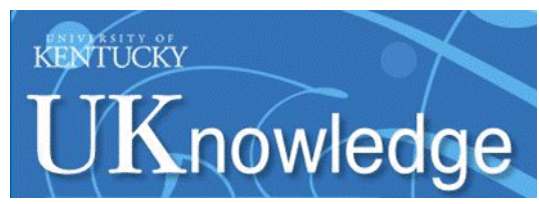

University of Kentucky

UKnowledge

$5-1-2020$

\title{
The $\boldsymbol{\beta} 3$-Adrenergic Receptor Agonist Mirabegron Improves Glucose Homeostasis in Obese Humans
}

\author{
Brian S. Finlin \\ University of Kentucky, bfinlin@email.uky.edu \\ Hasiyet Memetimin \\ University of Kentucky, m.hasiyet@uky.edu \\ Beibei Zhu \\ University of Kentucky, bzhu2@uky.edu \\ Amy L. Confides \\ University of Kentucky, amy.confides@uky.edu \\ Hemendra J. Vekaria \\ University of Kentucky, hemendravekaria@uky.edu
}

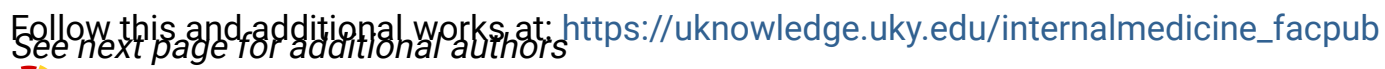

Part of the Endocrinology, Diabetes, and Metabolism Commons, Internal Medicine Commons, Neurology Commons, Physical Therapy Commons, and the Radiology Commons

Right click to open a feedback form in a new tab to let us know how this document benefits you.

\section{Repository Citation}

Finlin, Brian S.; Memetimin, Hasiyet; Zhu, Beibei; Confides, Amy L.; Vekaria, Hemendra J.; El Khouli, Riham H.; Johnson, Zachary R.; Westgate, Philip M.; Chen, Jianzhong; Morris, Andrew J.; Sullivan, Patrick G.; Dupont-Versteegden, Esther E.; and Kern, Philip A., "The ß3-Adrenergic Receptor Agonist Mirabegron Improves Glucose Homeostasis in Obese Humans" (2020). Internal Medicine Faculty Publications. 242. https://uknowledge.uky.edu/internalmedicine_facpub/242

This Article is brought to you for free and open access by the Internal Medicine at UKnowledge. It has been accepted for inclusion in Internal Medicine Faculty Publications by an authorized administrator of UKnowledge. For more information, please contact UKnowledge@lsv.uky.edu. 


\section{The $\boldsymbol{\beta} 3$-Adrenergic Receptor Agonist Mirabegron Improves Glucose Homeostasis in Obese Humans}

\section{Digital Object Identifier (DOI)}

https://doi.org/10.1172/JCl134892

\section{Notes/Citation Information}

Published in The Journal of Clinical Investigation, v. 130, no. 5.

Copyright $(2020$ American Society for Clinical Investigation

The copyright holder has granted the permission for posting the article here.

\section{Authors}

Brian S. Finlin, Hasiyet Memetimin, Beibei Zhu, Amy L. Confides, Hemendra J. Vekaria, Riham H. El Khouli, Zachary R. Johnson, Philip M. Westgate, Jianzhong Chen, Andrew J. Morris, Patrick G. Sullivan, Esther E. Dupont-Versteegden, and Philip A. Kern 


\section{The Journal of Clinical Investigation}

\section{The $\beta 3$-adrenergic receptor agonist mirabegron improves}

glucose homeostasis in obese humans

Brian S. Finlin, ... , Esther E. Dupont-Versteegden, Philip A. Kern

J Clin Invest. 2020;130(5):2319-2331. https://doi.org/10.1172/JCl134892.

\section{Clinical Medicine Clinical trials Metabolism}

\section{Graphical abstract}

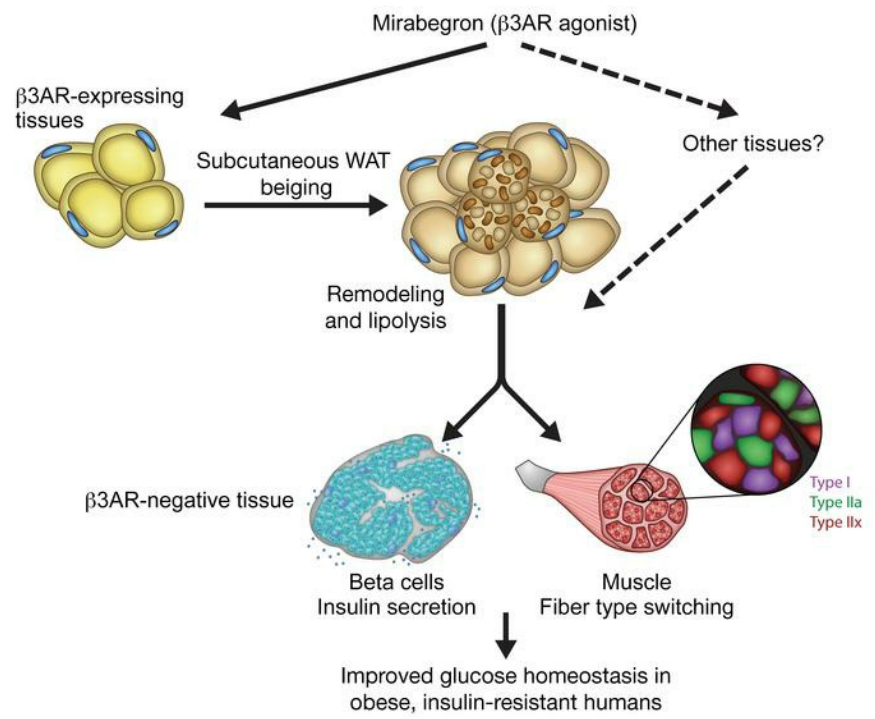

Find the latest version: 


\title{
The $\beta 3$-adrenergic receptor agonist mirabegron improves glucose homeostasis in obese humans
}

\author{
Brian S. Finlin, ${ }^{1,2}$ Hasiyet Memetimin,, ${ }^{1,2}$ Beibei Zhu, ${ }^{1,2}$ Amy L. Confides, ${ }^{3,4}$ Hemendra J. Vekaria, ${ }^{5}$ Riham H. El Khouli, ${ }^{6}$ \\ Zachary R. Johnson, ${ }^{1,2}$ Philip M. Westgate, ${ }^{7}$ Jianzhong Chen, ${ }^{8,9}$ Andrew J. Morris, ${ }^{8,9}$ Patrick G. Sullivan, ${ }^{5}$ \\ Esther E. Dupont-Versteegden, ${ }^{3,4}$ and Philip A. Kern ${ }^{1,2}$ \\ 1Division of Endocrinology, Department of Internal Medicine, and ${ }^{2}$ Barnstable Brown Diabetes and Obesity Center, College of Medicine, ${ }^{3}$ Department of Physical Therapy, College of Health Sciences, ${ }^{4}$ Center \\ for Muscle Biology, ${ }^{5}$ Spinal Cord and Brain Injury Research Center, and ${ }^{6}$ Department of Radiology, College of Medicine, ${ }^{7}$ College of Public Health, and ${ }^{8}$ Division of Cardiovascular Medicine, Department of \\ Internal Medicine, College of Medicine, University of Kentucky, Lexington, Kentucky, USA. 'Lexington Veterans Affairs Medical Center, Lexington, Kentucky, USA.
}

BACKCROUND. Beige adipose tissue is associated with improved glucose homeostasis in mice. Adipose tissue contains $\beta 3-$ adrenergic receptors ( $\beta 3$-ARs), and this study was intended to determine whether the treatment of obese, insulin-resistant humans with the $\beta 3-A R$ agonist mirabegron, which stimulates beige adipose formation in subcutaneous white adipose tissue (SC WAT), would induce other beneficial changes in fat and muscle and improve metabolic homeostasis.

METHODS. Before and after $\beta 3-A R$ agonist treatment, oral glucose tolerance tests and euglycemic clamps were performed, and histochemical analysis and gene expression profiling were performed on fat and muscle biopsies. PET-CT scans quantified brown adipose tissue volume and activity, and we conducted in vitro studies with primary cultures of differentiated human adipocytes and muscle.

RESULTS. The clinical effects of mirabegron treatment included improved oral glucose tolerance $(P<0.01)$, reduced hemoglobin A1c levels $(P=0.01)$, and improved insulin sensitivity $(P=0.03)$ and $\beta$ cell function $(P=0.01)$. In SC WAT, mirabegron treatment stimulated lipolysis, reduced fibrotic gene expression, and increased alternatively activated macrophages. Subjects with the most SC WAT beiging showed the greatest improvement in $\beta$ cell function. In skeletal muscle, mirabegron reduced triglycerides, increased the expression of PPAR $\gamma$ coactivator $1 \alpha(P C C 1 A)(P<0.05)$, and increased type I fibers $(P<0.01)$. Conditioned media from adipocytes treated with mirabegron stimulated muscle fiber $P C C 1 A$ expression in vitro $(P<0.001)$.

CONCLUSION. Mirabegron treatment substantially improved multiple measures of glucose homeostasis in obese, insulinresistant humans. Since $\beta$ cells and skeletal muscle do not express $\beta 3$-ARs, these data suggest that the beiging of SC WAT by mirabegron reduces adipose tissue dysfunction, which enhances muscle oxidative capacity and improves $\beta$ cell function.

TRIAL REGISTRATION. Clinicaltrials.gov NCT02919176.

FUNDING. NIH: DK112282, P30GM127211, DK 71349, and Clinical and Translational science Awards (CTSA) grant UL1TR001998.

\section{Introduction}

The $\beta 3$-adrenergic receptor ( $\beta 3$-AR) has long been a therapeutic target to combat obesity and metabolic disease. Since the $\beta 3-\mathrm{AR}$ is present in adipose tissue, but not in most other tissues, an agonist would activate thermogenesis in adipose tissue, stimulating lipid oxidation and glucose consumption to produce heat $(1,2)$ without causing cardiovascular side effects. Much initial work has been performed in rodents, which maintain brown

\section{Related Commentary: p. 2180}

Authorship note: BSF and HM contributed equally to this work. Conflict of interest: The authors have declared that no conflict of interest exists. Copyright: () 2020, American Society for Clinical Investigation. Submitted: November 8, 2019; Accepted: January 14, 2020; Published: March 23, 2020 Reference information: J Clin Invest. 2020;130(5):2319-2331. https://doi.org/10.1172/JCl134892. adipose tissue (BAT) throughout adulthood to defend against cold; indeed, treatment of rodents with $\beta 3$-AR agonists activates BAT, resulting in increased energy expenditure, weight loss, and improved glucose and lipid metabolism (3-5). In addition to stimulating white adipose tissue (WAT) lipolysis, it is now recognized that $\beta 3$-AR activation (or cold exposure) induces the formation of brown adipocytes in subcutaneous WAT (SC WAT) (6), which are now called beige adipocytes (7). Recent studies using tissue transplantation or genetic approaches have demonstrated that beige adipose tissue is associated with improved glucose and lipid homeostasis (8-12).

BAT is highly specialized and functions to generate heat in response to activation of the sympathetic nervous system through nonshivering thermogenesis (13). The adipocytes in BAT have higher levels of mitochondria than do those in WAT, and these mitochondria have high levels of uncoupling protein 1 (UCP1). Activation of the sympathetic nervous system stimulates lipoly- 


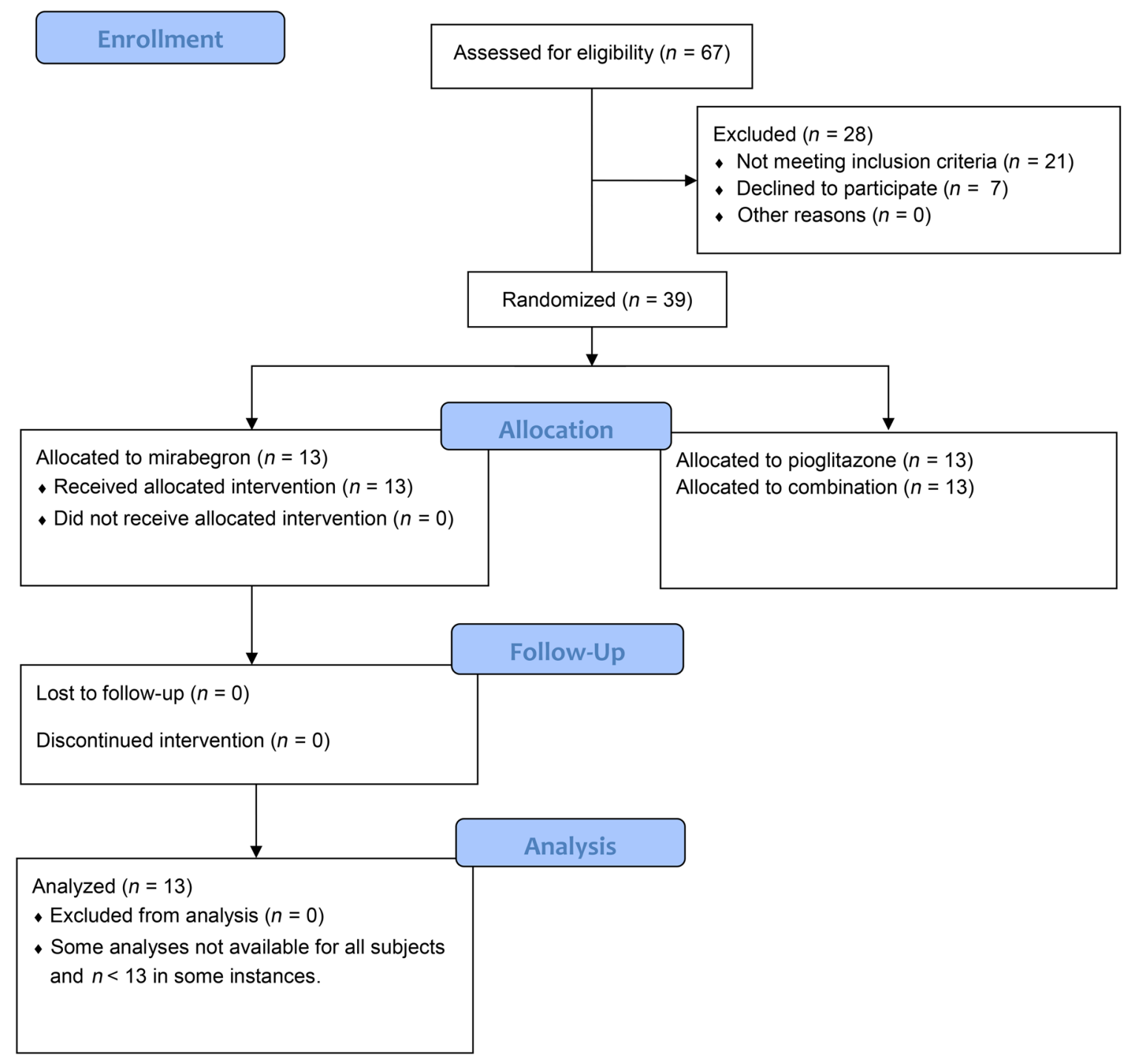

Figure 1. Flow chart of the study design and analysis of the research participants. Sixty-seven research subjects were assessed, and 39 were randomized into 3 drug treatment groups. The results for the subjects in the pioglitazone and combination therapy groups will be presented in a future publication. Thirteen subjects were in the mirabegron treatment group, and all completed the study.

sis and UCP1 activation in BAT, causing energy to be converted to heat and consuming lipids and glucose (13). BAT is present in adult humans (14-17) and is associated with improved insulin sensitivity and glucose homeostasis (recently reviewed in ref. 18). However, BAT diminishes with age and obesity, and there are questions about whether BAT activation is feasible therapeutically in the older, insulin-resistant human population that would be the logical target for drug treatment (19).

Beige adipocytes are unique, since they have a different developmental origin than brown adipocytes and are highly inducible by cold exposure or $\beta$-AR agonism (recently reviewed in ref. 20). Recent studies in mice have demonstrated that beige adipocytes can activate glycolytic pathways and improve glucose metabolism independently of UCP1 (21) and that futile metabolic cycles act in addition to UCP1-mediated uncoupled respiration $(21,22)$. Beiging is also associated with reduced adipose tissue fibrosis and adipose dysfunction $(11,23)$. These findings suggest that inducing beige adipose tissue may improve metabolic homeostasis by increasing the ability of SC WAT to function as a metabolic sink for glucose and lipids (20) or by reducing the WAT dysfunction that occurs with obesity $(24,25)$. Substantial beiging of human SC WAT has been demonstrated with cancer cachexia, burns, and conditions with high catecholamine levels (26-29), and we have demonstrated the induction of UCP1 and beige adipose markers in SC WAT in response to cold (30). However, to our knowledge, no study in humans has demonstrated a link between beige fat and glucose or lipid metabolism.

Mirabegron is a $\beta 3-\mathrm{AR}$ agonist that is FDA approved for the treatment of overactive bladder at a maximal dose of $50 \mathrm{mg} /$ day. In recent human studies, acute mirabegron treatment increased BAT activity and resting energy expenditure in lean humans with demonstrable baseline levels of BAT in a dose-dependent manner (31-33). We treated a cohort of human subjects who were older, obese, and insulin resistant with mirabegron for 12 weeks and observed that chronic mirabegron treatment stimulated SC WAT beiging (30). 


\section{Table 1. Baseline characteristics of study subjects and treatment responses}

\begin{tabular}{lccc} 
& \multicolumn{2}{c}{ Mirabegron $^{\mathrm{A}}$} & ${\text { P } \text { value }^{\mathrm{B}}}$ \\
& Before & After & \\
Number (Sex, male/female) & $13(2 / 11)$ & & \\
Age (yr) & $54.8 \pm 2.2$ & & \\
BMI (kg/m²) & $32.4 \pm 1.1$ & & \\
Weight (kg) & $88.0 \pm 2.6$ & $87.5 \pm 2.6$ & 0.34 \\
Systolic BP (mmHg) & $127.8 \pm 4.8$ & $125.5 \pm 3.5$ & 0.42 \\
Diastolic BP (mmHg) & $79.8 \pm 2.3$ & $81.4 \pm 2.6$ & 0.61 \\
Heart rate (beats/min) & $68.7 \pm 1.6$ & $70.7 \pm 1.8$ & 0.32 \\
Fasting glucose (mg/dL) & $97.0 \pm 2.8$ & $95.8 \pm 3.1$ & 0.49 \\
Fasting insulin ( $\mu \mathrm{U} / \mathrm{mL})$ & $13.2 \pm 2.7$ & $14.4 \pm 2.3$ & 0.4 \\
120-minute glucose (mg/dL) & $135.3 \pm 7.5$ & $109.8 \pm 6.4$ & $<0.01$ \\
HbA1c (\%) & $5.6 \pm 0.1$ & $5.4 \pm 0.1$ & 0.01 \\
TC (mg/dL) & $144.0 \pm 22.9$ & $130.1 \pm 18.0$ & 0.35 \\
Cholesterol (mg/dL) & $215.7 \pm 11.2$ & $205.7 \pm 11.6$ & 0.09 \\
HDL (mg/dL) & $56.6 \pm 5.1$ & $55.9 \pm 4.7$ & 0.71 \\
LDL (mg/dL) & $132.9 \pm 7.8$ & $125.4 \pm 8.6$ & 0.15
\end{tabular}

${ }^{A}$ Research participants were treated with mirabegron ( $50 \mathrm{mg} /$ day) for 12 weeks. Data indicate the mean $\pm \mathrm{SEM}(n=13)$. ${ }^{\mathrm{B}}$ Data were analyzed by a paired, 2-tailed Student's $t$ test.

Here, we present the results of extensive metabolic phenotyping of an expanded cohort of obese, insulin-resistant subjects before and after mirabegron treatment. We found that mirabegron treatment substantially improved glucose homeostasis and addressed many of the underlying mechanisms of insulin resistance, including improved insulin sensitivity and secretion and improved adipose tissue and skeletal muscle lipotoxicity and inflammation, yet had no effect on BAT in these older, obese individuals.

\section{Results}

Effect of mirabegron treatment on lipid and glucose homeostasis. A flow diagram showing the subject recruitment and study design is shown in Figure 1. We observed no significant change in body weight after treatment with mirabegron (Table 1). Consistent with this, mirabegron treatment did not change any measure of body composition (Supplemental Table 1; supplemental material available online with this article; https://doi.org/10.1172/JCI134892DS1) or resting energy expenditure (Supplemental Table 2). Mirabegron treatment did not significantly change plasma lipid levels, although there was a trend toward a reduction in total cholesterol (Table $1 ; P=0.09$ ). In addition, there were no reported side effects, in particular, no cardiovascular side effects such as palpitations or rapid pulse. Blood pressure and heart rate did not change with treatment (Table 1), consistent with previous studies (34).

We assessed glucose homeostasis with an oral glucose tolerance test (OGTT) and measurement of hemoglobin A1c (HbA1c) levels. Fasting blood glucose was not affected by mirabegron treatment (Table 1); however, mirabegron treatment significantly improved overall oral glucose tolerance $(P<0.01 ; n=12)$, as shown in Figure 2A. The glucose concentration 120 minutes after oral glucose ingestion was significantly lower after mirabegron treatment (Table $1 ; P<0.01$ ). A subset of subjects had impaired glucose tolerance, and their 120-minutes glucose levels decreased from $165 \mathrm{mg} / \mathrm{dL}$ to $120 \mathrm{mg} / \mathrm{dL}$ after treatment. Among the 13 subjects, 9 had prediabetes according to American Diabetes Association criteria (i.e., having any one of the following: fasting glucose levels of 100-125 mg/dL, 120-minute glucose levels of 140-199 mg/dL, or HbA1c between $5.7 \%$ and $6.4 \%$ ). Following mirabegron treatment for 12 weeks, 5 of the 9 subjects were no longer prediabetic (i.e., did not meet any of the criteria described above). Overall, these subjects did not have diabetes, and the mean HbA1c level of the group was $5.6 \% \pm 0.1 \%$ at baseline (Table 1 ). HbA1c was significantly lower after mirabegron treatment (Table $1 ; P=0.01$ ), consistent with the overall improvement in glucose tolerance.

Insulin sensitivity and pancreatic $\beta$ cell function. To determine the underlying mechanisms for the improved glucose tolerance, we evaluated insulin sensitivity and pancreatic $\beta$ cell function using the results obtained from both the OGTT and euglycemic clamp. Mirabegron treatment did not significantly change baseline insulin levels (Table 1), nor did it change the homeostatic model assessment of insulin resistance (HOMA-IR) or the Matsuda index, which are measures of insulin sensitivity derived from the results of the OGTT (33). However, the results from euglycemic clamps, which are the gold standard for measuring insulin sensitivity (35), revealed that mirabegron treatment consistently and significantly increased the glucose infusion rate (GIR) by approximately $12 \%$ (Figure $2 \mathrm{~B} ; P=0.03$ ). Using glucose and insulin values from the OGTT, we calculated the insulinogenic index as an indicator of unadjusted insulin secretion. Using the insulin sensitivity from the clamp and the insulinogenic index, we then calculated insulin secretion adjusted for insulin sensitivity to obtain the disposition index value (36). We found that mirabegron treatment significantly increased both the insulinogenic index (Figure 2C; $P=0.02$ ) and the disposition index (Figure 2D; $P=0.01$ ), indicating improved $\beta$ cell function. We measured insulin levels during the OGTT (Supplemental Figure 1A) and the euglycemic clamps (Supplemental Figure 1B) and did not find evidence that mirabegron changed insulin clearance rates. Finally, although there was no significant weight loss, some individuals did weigh less after mirabegron treatment. We therefore performed analyses to determine whether the percentage of weight change influenced insulin sensitivity, but found no significant relationship $(P=0.55)$. Together, these results indicate that the improvement in glucose tolerance was the result of both increased $\beta$ cell function and improved insulin sensitivity.

Beige adipose tissue and BAT. The $\beta 3$-AR has a restricted tissue distribution, with expression reported in white and brown adipocytes and bladder smooth muscle cells. It is thus possible that the effects on insulin sensitivity and $\beta$ cell function observed in our study were not direct but rather the result of SC WAT beiging or brown fat induction. Indeed, the activity of both beige adipose tissue and BAT is associated with improved glucose homeostasis and postulated to involve the secretion of "batokines" to communicate with peripheral tissues (20). In previous studies, mirabegron was demonstrated to cause an acute increase in BAT activity in young, lean male subjects with detectable levels of BAT at baseline and was more effective at the higher dose of $200 \mathrm{mg}$, which is 4 times the FDA-approved dose $(31,32)$. To determine whether chronic mirabegron treatment $(50 \mathrm{mg} /$ day) increased BAT activity in 
A

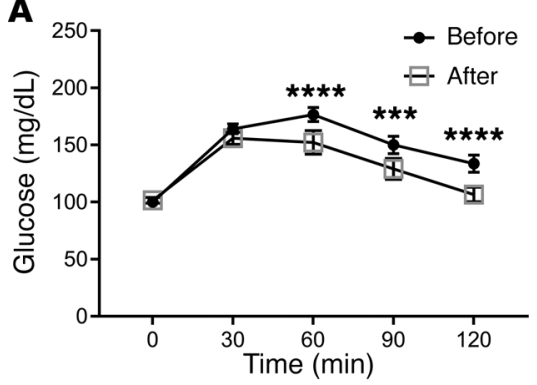

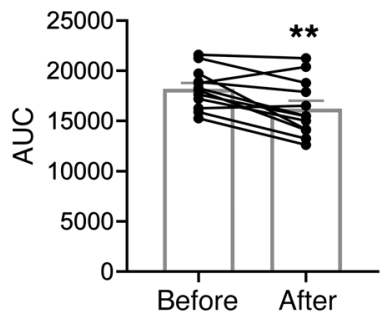

B

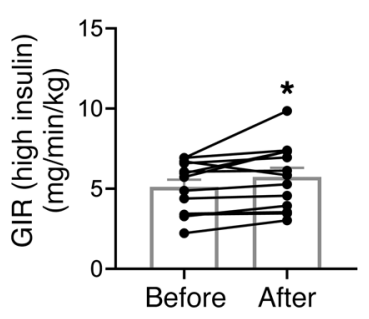

C

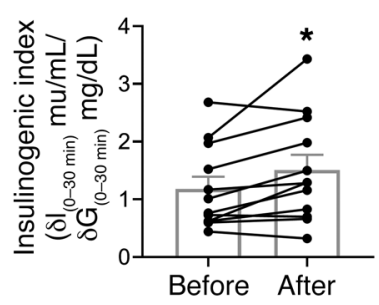

D

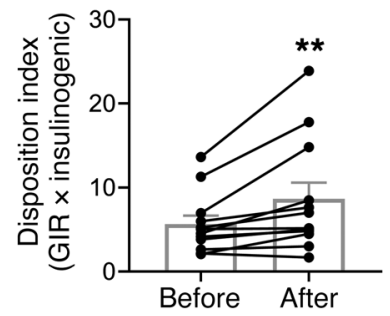

E

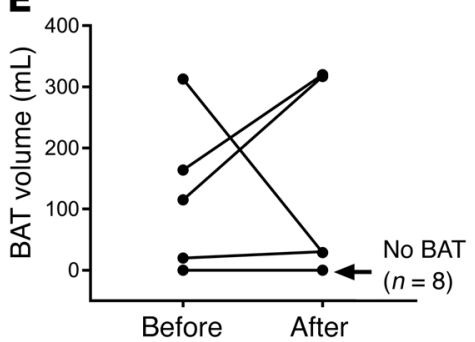

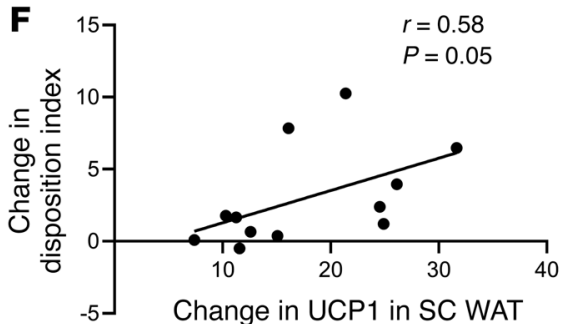

Figure 2. Mirabegron treatment improves glucose homeostasis in obese, insulin-resistant subjects. Subjects were treated with mirabegron (50 mg/ day) for 12 weeks. OCTTs and euglycemic clamps were performed at baseline and after treatment. (A) OCTT results and AUC for all subjects ( $n=12$ ). Data indicate the mean \pm SEM. OCTT data were analyzed by a 2 -way, repeated-measures ANOVA $\left({ }^{* *} P<0.001\right.$ and $\left.{ }^{* * * *} P<0.0001\right)$; AUC data were analyzed by a paired, 2-tailed Student's $t$ test ( $\left.{ }^{*} P<0.01\right)$. (B) Euglycemic clamping was performed at an insulin infusion rate of 1.0 mU/kg/min, and the GIR was determined before and after treatment. Data indicate the mean \pm SEM $(n=13)$ and were analyzed by a paired, 2 -tailed Student's $t$ test ( $P<0.05)$. (C) The insulinogenic index was determined from the results of the oral glucose tolerance test. Data indicate the mean \pm SEM ( $n=12$ ) and were analyzed by a paired, 2-tailed Student's $t$ test ( $\left.{ }^{*} P<0.05\right)$. (D) The disposition index is the product of (C) insulin sensitivity and (D) the insulinogenic index. Data indicate the mean \pm SEM $(n=12)$. ${ }^{*} P<0.01$, by paired, 2-tailed Student's $t$ test. (E) BAT volume was quantified by PET-CT scans before and after treatment. Eight subjects had no BAT at baseline, and BAT did not increase after treatment of these subjects. (F) The change in SC WAT beiging was calculated as the difference in UCP1 protein expression before and after treatment. The change in the disposition index (D) and the change in UCP1 in SC WAT were analyzed by regression analysis. The Spearman's correlation coefficient and $P$ value are indicated in $\mathbf{F}$.

older, insulin-resistant individuals, we performed cold-stimulated PET-CT before and after mirabegron treatment. As shown in Figure 2E, mirabegron treatment did not increase BAT volume. Notably, 8 of the subjects had no demonstrable BAT at baseline, and there was no increase in BAT in these subjects following mirabegron treatment. There was also no increase in any measure of glucose uptake by BAT (Supplemental Table 3).

We previously observed that mirabegron treatment significantly induced beiging of SC WAT in 6 subjects (30) who were among the 13 subjects in this report. Consistent with that study, we found that mirabegron treatment increased protein expression of the beige adipose markers UCP1 (2.4-fold, $P<0.0001)$, transmembrane protein 26 (TMEM26) (4.2-fold, $P<0.001)$, and cell deathinducing DFFA-like effector A (CIDEA) $(2.4$-fold, $P<0.01)$ in SC WAT within the entire cohort $(n=13)$. Since mirabegron treatment increased beige fat, we determined whether the change in adipose beiging correlated with the changes in insulin sensitivity or the disposition index. This analysis revealed that the change in UCP1 protein expression in SC WAT significantly correlated with the change in the disposition index, indicating a possible beneficial relationship between beige fat and pancreatic $\beta$ cell function (Figure $2 \mathrm{~F} ; P$ $=0.05$ ). Although we found a relationship between the change in UCP1 protein expression in SC WAT and the insulinogenic index, this change did not reach statistical significance $(r=0.46 ; P=0.12)$. The change in UCP1 protein expression in SC WAT did not significantly correlate with the change in the $\operatorname{GIR}(P=0.27)$.
Systemic inflammation and skeletal muscle lipotoxicity. Inflammation and lipotoxicity are both implicated in the development of insulin resistance and reduced $\beta$ cell function in the context of obesity in humans. We measured plasma levels of adiponectin, high-molecular-weight (HMW) adiponectin, TNF- $\alpha$, and monocyte chemoattractant protein 1 (MCP1). Plasma levels of TNF- $\alpha$, MCP1, or total or HMW adiponectin did not change after mirabegron treatment (Supplemental Table 4). We measured triglyceride (TG), diacylglyceride (DAG), and ceramide levels in vastus lateralis muscle biopsies to determine whether mirabegron treatment reduced lipotoxicity. Mirabegron treatment caused a slight reduction in TG levels (Figure 3A; $P=0.04$ ) but did not reduce levels of the lipotoxic lipids ceramide or DAG (Figure 3, B and C).

We investigated mechanisms for the reduction in muscle TG. PPAR $\gamma$ coactivator $1 \alpha(\mathrm{PGC} 1 \alpha)$ is part of a transcriptional network that regulates muscle fiber type determination and promotes fatty acid oxidation, mitochondrial biogenesis, and type I fiber formation (37, 38). PGC1A mRNA expression was significantly increased in muscle following mirabegron treatment (Figure 3D; $P<0.05$ ). Both mitochondrial transcription factor A (TFAM1) and cyclooxygenase IV (COX IV) were increased after mirabegron treatment (Figure 3, E and F; $P<0.01$ ), consistent with the induction of $P G C 1 A$. In addition to $P G C 1 A$, we measured the mRNA expression of perilipin 5 (PLIN5), which is associated with increased insulin sensitivity. This increase is thought to be due to the ability of PLIN5 to promote fatty acid oxidation (39). We observed that 
A
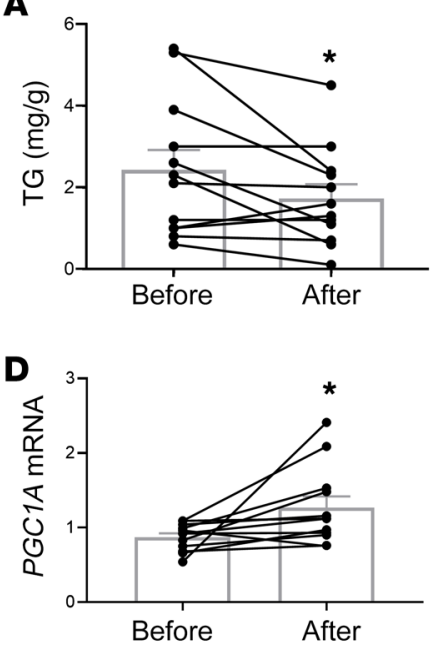

B

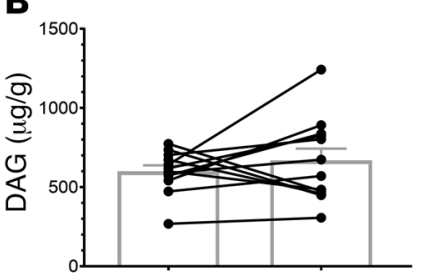

Before

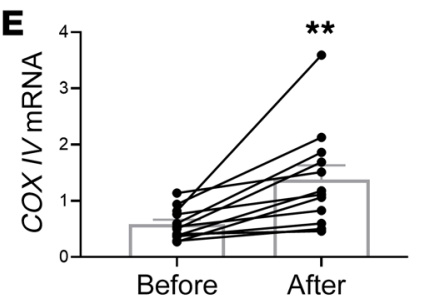

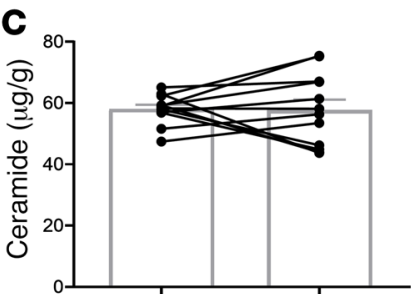

Before
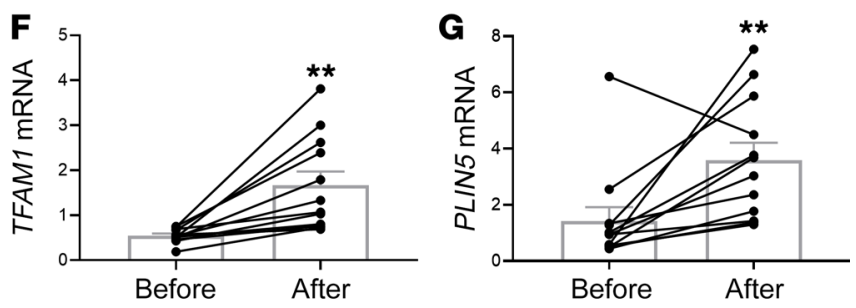

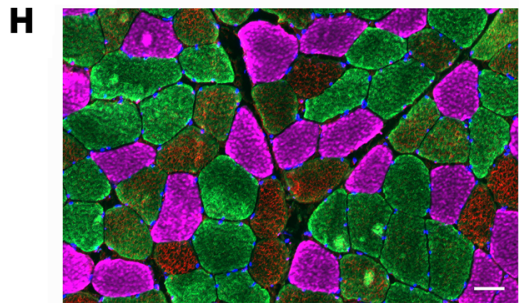

Before

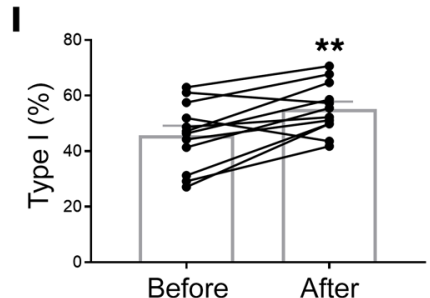

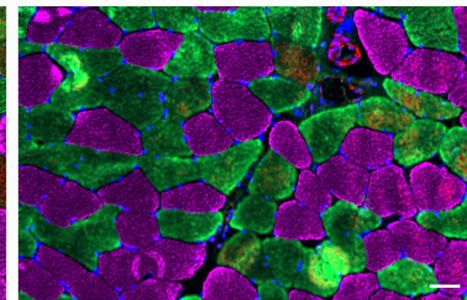

After
Type I

Type lla

Type IIx

Figure 3. Mirabegron treatment reduces skeletal muscle TGs, but not toxic lipids, and promotes fiber-type switching to type I fibers. Lipids were extracted from skeletal muscle obtained from vastus lateralis biopsies and the levels of the indicated lipids were measured as described in Methods. The levels of (A) TG, (B) DAG, and (C) ceramide before and after mirabegron treatment were determined. (D-G) mRNA expression of genes in muscle was determined by real-time RT-PCR. (H) Representative images of muscle stained for MyHC I, MyHC Ila, and MyHC IIx before and after mirabegron treatment. Scale bars: $50 \mu \mathrm{m}$. (I-L) Quantification of type I, type Ila, and type Ilx fibers. Data are expressed as the percentage of total fibers and indicate the mean \pm SEM $(n=12-13)$. ${ }^{*} P<0.05$ and ${ }^{* *} P<0.01$, by paired, 2 -tailed Student's $t$ test.

PLIN5 was upregulated with mirabegron treatment (Figure 3G; $P<$ 0.01). A possible explanation for these changes in gene expression is that mirabegron treatment changed the fiber type composition of skeletal muscle; therefore, we quantified type I, type IIa, type IIx, and type IIa /IIx fibers. As shown in Figure 3, H-L, the percentage of type I fibers significantly increased $(P<0.01)$, the percentage of type IIa fibers significantly decreased $(P<0.05)$, and neither type IIx nor type IIa/IIx fibers changed following mirabegron treatment.

Real-time reverse transcription PCR (RT-PCR) revealed no mRNA expression of the $\beta 3$-AR in muscle; as expected, we were able to detect mRNA expression of the $\beta 2$-AR (data not shown). Activation of the $\beta 2$-AR causes skeletal muscle hypertrophy (40); however, we did not detect an increase in the size of the muscle fibers (data not shown), suggesting that mirabegron did not cause activation of $\beta 2$-ARs at the dose used in this study. Thus, these changes in skeletal muscle induced by mirabegron treatment are probably caused by an indirect mechanism that is possibly related to SC WAT beiging. To test this concept in vitro, we prepared conditioned medium (CM) from untreated adipocytes and from adipocytes treated with mirabegron for 16 hours. We then treated differentiated human myotubes with mirabegron, CM from differentiated human adipocytes, or CM from adipocytes treated with mirabegron for 16 hours. We found that CM from untreated adipocytes did not induce PGC1A mRNA expression in myotubes; however, myotubes that were incubated with $\mathrm{CM}$ from adipocytes that were treated with mirabegron had significantly increased levels of $P G C 1 A$ expression (Figure $4 ; P<0.001$ ), suggesting that mirabegron treatment caused adipocytes to release a factor that induced PGC1A mRNA expression in muscle. As an additional control to account for any effect of mirabegron on myotubes, we harvested $\mathrm{CM}$ from adipocytes and then added mirabegron to it and treat- 


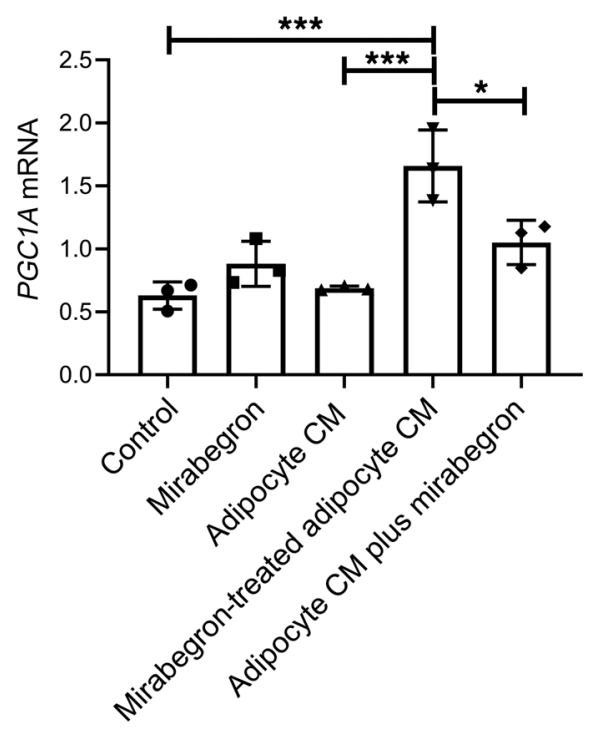

ed myotubes with this medium. CM from adipocytes treated with mirabegron induced higher PGC1A expression in myotubes than did media in which mirabegron was added after harvesting the CM from adipocytes. (Figure $4 ; P<0.05$ ).

Adipose tissue remodeling. We have previously reported that phospho-hormone-sensitive lipase (HSL) is increased after mirabegron treatment (30), suggesting that mirabegron stimulates cAMP signaling in adipocytes. To determine whether mirabegron treatment stimulates lipolysis, we measured nonesterified fatty acids (NEFAs) and glycerol in the CM from adipose explants from subjects before and after treatment. We found that glycerol was significantly higher in the medium of adipose tissue after mirabegron treatment (Figure 5A; $P<0.01$ ) and that plasma NEFA levels were higher after mirabegron treatment (Figure 5B; $P<0.05$ ). Elevated plasma NEFA levels are thought to contribute to ectopic lipid accumulation and inhibition of insulin receptor signaling in the liver and skeletal muscle. Acute exposure of $\beta$ cells to NEFAs stimulates insulin secretion, but chronic exposure causes lipotoxicity and inhibits insulin secretion (41-43). As described above, ceramide and DAG levels in skeletal muscle were not elevated, despite the increase in plasma NEFAs. This raises the interesting possibility that $\beta 3$-AR stimulation of beiging induces compensatory mechanisms, such as the fiber-type switching to the more oxidative type I muscle fibers described above, allowing for improved peripheral tissue function even in the presence of increased plasma NEFA levels.

To gain further insight into the effects of mirabegron on adipose tissue, we analyzed SC WAT gene expression before and after treatment using the NanoString nCounter Multiplex system. The panel of 160 genes consisted of adipokines, cytokines, immune cell markers, and genes involved in adipocyte function, angiogenesis, and fibrosis (Supplemental Table 5). Fatty acid-binding protein 4 (FABP4) mRNA expression was significantly induced following mirabegron treatment (Table 2; $P<0.01$ ). FABP4, a lipid chaperone, is regulated by fatty acids (44) and is probably induced by mirabegron treatment in response to increased lipolysis. Collagen 6 and elastin as well as metalloproteinases and tissue inhibitors of metalloproteinases (TIMPs) were significantly changed by mirabegron
Figure 4. CM isolated from adipocytes treated with mirabegron induces PGC1 $\alpha$ expression in human myotubes in vitro. Differentiated human adipocytes, with or without $100 \mathrm{nM}$ mirabegron treatment for 16 hours, and the CM was isolated as described in Methods. An additional control was made by adding mirabegron to the CM after the CM was isolated from the adipocytes. The human myotubes were then incubated with $0.025 \%$ DMSO (Control), mirabegron (25 nM), adipocyte CM (25\%), mirabegron-treated adipocyte CM (25\%), or adipocyte CM plus mirabegron (25\%). The final concentration of mirabegron was $25 \mathrm{nM}$. Data indicate the mean \pm SEM $(n=3) .{ }^{*} P<0.05$ and ${ }^{* *} P<0.001$, by 1 -way ANOVA with Tukey's multiple comparisons test.

treatment, and most of these were lower after treatment (Table 2), suggesting a reduction in extracellular matrix (ECM) remodeling. The mRNA expression levels of 2 genes encoding secreted proteins with known effects on insulin resistance were changed: fibroblast growth factor 21 (FGF21), which has overall beneficial effects on metabolic homeostasis, was induced by mirabegron treatment, and resistin, which is associated with insulin resistance, was suppressed by mirabegron treatment. Retinol-binding protein 4 (RBP4) mRNA expression was elevated; RBP4 is a secreted protein that is postulated to promote local adipose tissue inflammation; however, we did not find increased inflammatory gene expression. Also, mirabegron treatment did not affect the expression of any of the $\beta$-ARs (Supplemental Figure 2). Overall, mirabegron treatment yielded many changes in SC WAT gene expression that indicated a shift toward an improvement in adipose tissue function. Finally, we found that mirabegron did not induce changes in the plasma levels of resistin, FGF21, adipsin, or leptin proteins (Supplemental Table 4).

To assess adipose tissue inflammation, we measured adipose tissue macrophage polarization in response to mirabegron. The numbers of $\mathrm{CD} 163^{+} \mathrm{CD} 68^{+}$macrophages were increased in SC

\section{Table 2. Changes in SC WAT gene expression caused by mirabegron}

$\begin{array}{lccc}\text { Cene symbol }^{\mathrm{A}} & \text { Function } & \text { Fold change } & \boldsymbol{P}_{\text {value }}{ }^{\mathrm{B}} \\ \text { FABP4 } & \text { Fatty acid metabolism } & 1.22 & <0.01 \\ \text { RBP4 } & \text { Secreted factor } & 1.24 & <0.01 \\ \text { TIMP2 } & \text { ECM } & 0.85 & <0.01 \\ \text { COL6A1 } & \text { ECM } & 0.89 & 0.02 \\ \text { Angiopoietin 3 } & \text { Angiogenesis } & 0.81 & 0.03 \\ \text { DGAT2 } & \text { Lipid metabolism } & 1.24 & 0.03 \\ \text { Angiopoietin 4 } & \text { Angiogenesis } & 1.19 & 0.03 \\ \text { MMP2 } & \text { ECM } & 0.69 & 0.03 \\ \text { PPARG2 } & \text { Transcription factor } & 1.18 & 0.04 \\ \text { Resistin } & \text { Secreted factor } & 0.67 & 0.04 \\ \text { FCF21 } & \text { Secreted factor } & 1.21 & 0.04 \\ \text { CERS6 } & \text { Lipid metabolism } & 1.11 & 0.04 \\ \text { PKCA } & \text { Kinase } & 0.87 & 0.05 \\ \text { Elastin } & \text { ECM } & 0.76 & 0.05 \\ \text { CM-CSF } & \text { Cytokine } & 0.83 & 0.05 \\ \text { MMP14 } & \text { ECM } & 0.88 & 0.05\end{array}$

${ }^{A}$ Research participants were treated with mirabegron ( $50 \mathrm{mg} /$ day) for 12 weeks, and gene expression was measured at baseline and after treatment. Data represent mean \pm SEM $(n=13)$. ${ }^{B}$ Data were analyzed by a paired, 2 -tailed Student's $t$ test. Additional information is available in Supplemental Table 5. 
A

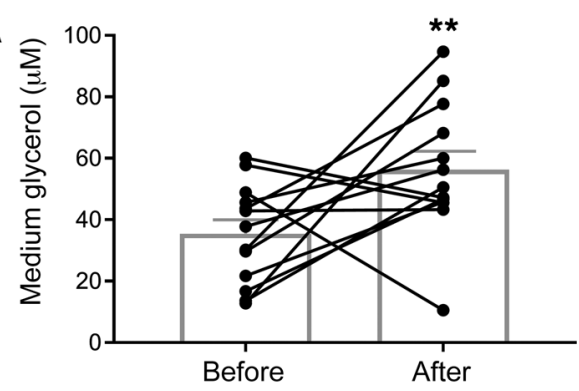

B

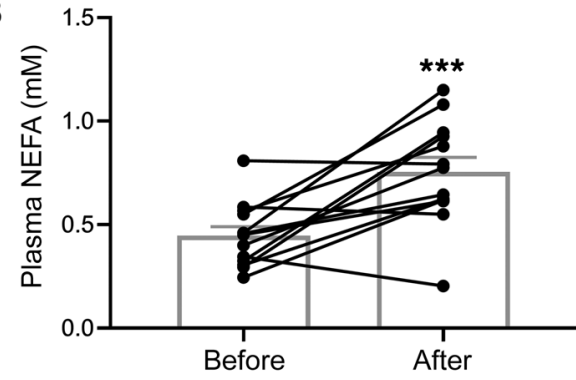

Figure 5. Mirabegron treatment stimulates lipolysis. (A) Adipose tissue $(0.5 \mathrm{~g})$ from the SC WAT biopsy was placed in medium and kept at $37^{\circ} \mathrm{C}$ for 1 hour, and the level of glycerol was determined in the adipose tissue explant CM before and after treatment. (B) Plasma NEFA levels were determined before and after treatment. Data indicate the mean $\pm \operatorname{SEM}(n=13) .{ }^{*} P<$ 0.01 and ${ }^{* *} P<0.001$, by paired, 2 -tailed Student's $t$ test.

WAT after mirabegron treatment $(P<0.05)$, but the numbers of $\mathrm{CD} 86^{+} \mathrm{CD} 68^{+}$macrophages were not changed (Figure 6, A and B). This result indicates a shift toward M2 macrophage polarization and suggests reduced adipose dysfunction, consistent with the results of the gene expression analysis. Finally, we found that UCP1 was often present as punctate staining in small cells between the large, unilocular adipocytes. UCP1 staining colocalized with $\mathrm{CD} 163$, and $\mathrm{UCP} 1^{+} \mathrm{CD}_{163}{ }^{+}$cells significantly increased in SC WAT after mirabegron treatment (Figure 6, C and D; $P$ $<0.001$ ). These results suggest that UCP1 is not only present in beige adipocytes but is also expressed in alternatively activated macrophages, which may play an important role in beige adipose tissue in humans.

SC WAT mitochondrial bioenergetics. We previously demonstrated that repeated cold exposure increases mitochondrial uncoupling in SC WAT (30). Using similar methods, we examined the bioenergetic profiles of purified mitochondria from SC WAT before and after mirabegron treatment by measuring state 3 and state 4 respiration, uncoupling activity, and maximal respiration. The bioenergetic profiles before and after treatment with mirabegron are shown in Figure 7, A and B. To measure UCP1-driven uncoupling, we calculated the difference between the oxygen consumption rates (OCRs) obtained during the presence of oligomycin and then free fatty acids (FFAs), which were subsequently added to stimulate uncoupling. Although mirabegron induced UCP1 protein expression, it did not increase uncoupled respiration. However, respiration in the presence of oligomycin (state 4) was lower after mirabegron treatment (Figure 7, A and B). As part of our experimental protocol, we added BSA after the addition of FFAs to sequester the FFAs, which demonstrated the effects of FFAs on uncoupling. The OCR after BSA addition was lower than the OCR after oligomycin addition, both before (Figure 7A) and after treatment (Figure 7B), and this difference (Oligo - BSA) likely reflects stimulation of uncoupled respiration by endogenous FFAs or ROS, both of which are sequestered by BSA. As shown in Figure 7C, Oligo - BSA was significantly lower after mirabegron treatment $(P<0.05)$. The reduction in uncoupled respiration (Oligo - BSA) and the increase in metabolic efficiency likely reflect a beneficial effect of mirabegron treatment on SC WAT.

Summary and conclusions. Mirabegron treatment improved glucose homeostasis in obese, insulin-resistant subjects. The underlying mechanisms involve both increased insulin sensitivity and $\beta$ cell function. We found that mirabegron treatment caused an increase in type I fibers in skeletal muscle and had numerous positive effects on skeletal muscle gene expression that likely arose from increased PGC1 $\alpha$ expression. These changes in muscle would be predicted to increase insulin sensitivity and fatty acid oxidation. However, we were unable to detect $A D R B 3 \mathrm{mRNA}$ expression in muscle, and the $\beta 3-\mathrm{AR}$ is also not expressed by $\beta$ cells (45). Thus, the effects on both $\beta$ cells and muscle are probably indirect and caused by the numerous changes to SC WAT including beiging, adipose tissue remodeling, and reduced inflammation. Mirabegron did not induce BAT, and we found no evidence of increased uncoupled respiration or lipid oxidation, even though mirabegron treatment induced UCP1 protein expression in SC WAT. Furthermore, the beneficial physiological effects of mirabegron treatment on glucose metabolism, insulin sensitivity, and $\beta$ cell function occurred paradoxically in the presence of increased plasma NEFA levels. The increased NEFA levels did not result in muscle lipotoxicity, which may be due to an increase in type I fibers, suggesting that protective mechanisms are induced by mirabegron treatment. This human study supports the previously proposed concept that adipose beiging has beneficial effects on glucose metabolism, which can be UCP1 independent (21).

\section{Discussion}

Considerable evidence points to BAT and beige adipose tissue as both a metabolic sink for glucose and lipids and a source of "batokines" that affect peripheral tissues (20). $\beta 3$-AR activation is currently the best-known pharmacological approach for stimulating brown and beige fat, and the $\beta 3$-AR agonist mirabegron was demonstrated to be effective in activating BAT and inducing beige adipose in humans (30-32). The current study differs from previous ones in several important ways. In this study, we targeted an at-risk population: individuals with metabolic syndrome or prediabetes rather than lean, healthy subjects. In addition, the standard FDA-approved dose of mirabegron was used for 12 weeks and was very well tolerated, whereas previous studies used this $\beta 3$ agonist only acutely and sometimes in doses that had cardiovascular side effects. Finally, this study focused on both SC WAT beiging as well as BAT, along with a mechanistic examination of adipose and muscle.

The main findings of this study are that mirabegron treatment improved glucose metabolism in the absence of weight loss and that this improvement in glucose metabolism was clinically significant. Indeed, a recent study suggests that in a population of nondiabetic subjects, changes in glucose tolerance or HbA1c, as observed in our study, would be expected with a 

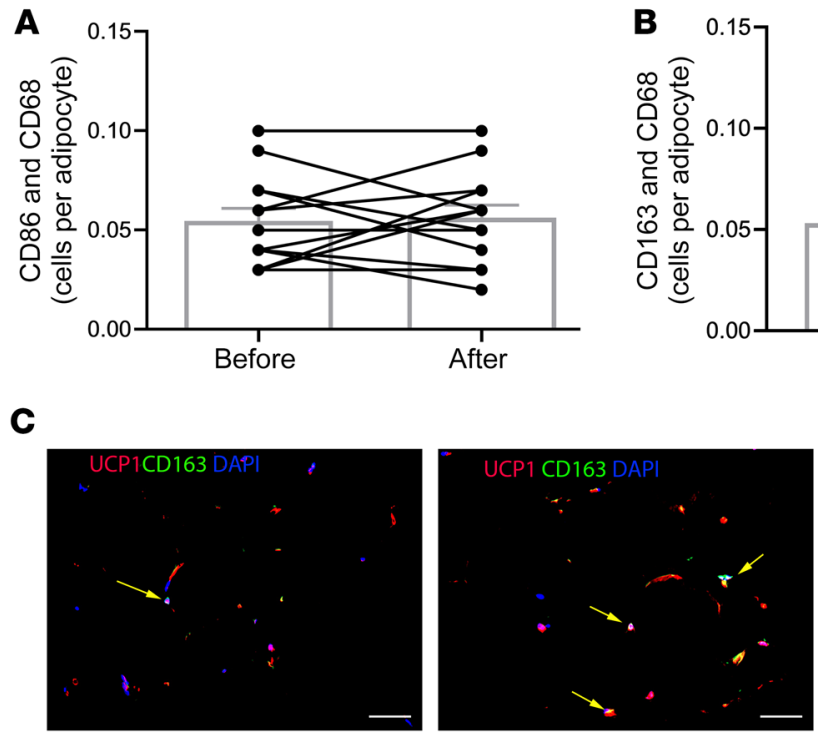
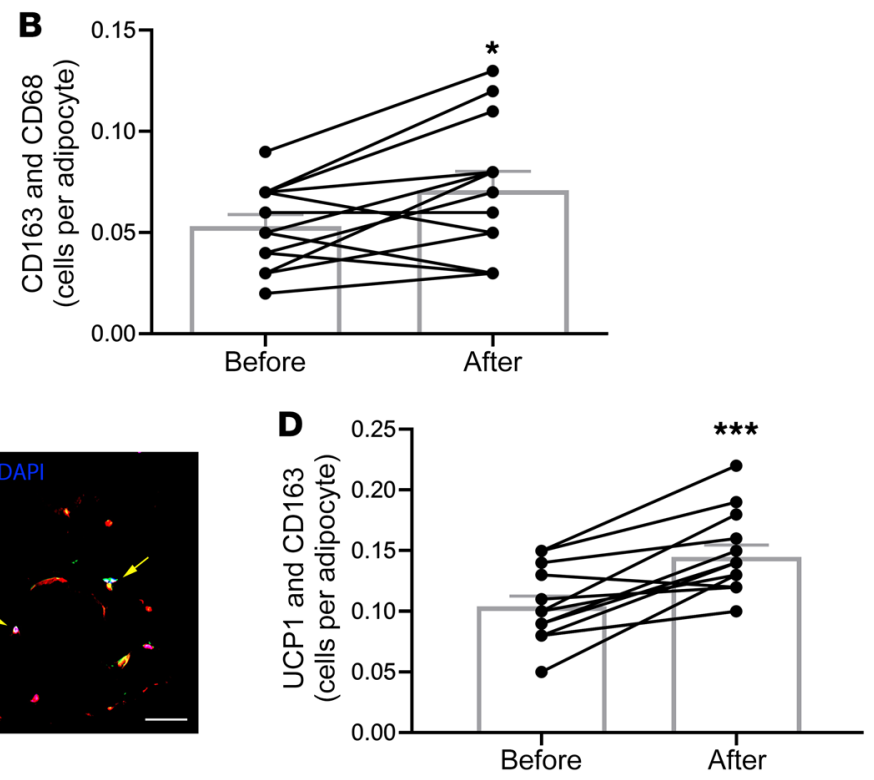

Figure 6. Mirabegron treatment increases alternatively activated macrophages in SC WAT. To characterize macrophage polarization, adipose tissue sections were doubly stained for (A) CD86 and CD68 (M1) and (B) CD163 and CD68 (M2). (C) UCP1 was present in CD163 ${ }^{+}$cells in SC WAT. Yellow arrows point to UCP1+CD163+DAPI+ cells (scale bar: $50 \mu \mathrm{m}$ ). (D) UCP1+CD163+ cells were quantified in SC WAT before and after mirabegron treatment. Data indicate the mean \pm SEM $(n=13)$. ${ }^{*} P<0.05$ and ${ }^{* *} P<0.001$, by paired, 2 -tailed Student's $t$ test.

weight loss of more than $16 \%$ (46). Finally, our observations that mirabegron treatment had beneficial effects (an increase in both insulin sensitivity and $\beta$ cell function) on cell types that do not express the $\beta 3$-AR indicate that SC WAT beiging affects the function of peripheral tissues. Indeed, we identified a significant correlation between the change in SC WAT UCP1 expression and the change in the disposition index, but not with the change in insulin sensitivity. Furthermore, we were not able to identify plasma adipokines that changed in response to mirabegron treatment. This finding suggests that mirabegron treatment may have had only a modest effect on adipose tissue or caused other tissues that express the $\beta 3$-AR to influence peripheral tissues involved in glucose homeostasis. Indeed, a recent study involving lean humans demonstrated that mirabegron influences bile acid metabolism (32).

The effects of mirabegron on metabolism have recently been studied in mice, and there are notable differences between those studies $(5,47)$ and this one in humans. For instance, treatment of $A p o E^{-/-}$mice with mirabegron increased total and LDL cholesterol and promoted atherosclerosis (47), but the current study in humans found a trend toward decreased cholesterol and no evidence for an increase in LDL. In another study in mice, mirabegron improved insulin sensitivity and glucose tolerance but also induced weight loss and significantly increased BAT activity (5). In this human study, mirabegron treatment clearly affected the function of peripheral tissues involved in glucose homeostasis, but there was no weight loss, and BAT activation was not a factor; the presence of BAT in mice, but not obese humans, likely accounts for the different responses. Other studies have demonstrated that both cold and mirabegron are capable of inducing BAT recruitment in humans $(31,32,48-51)$. However, the participants in those studies were usually lean and younger than the subjects in this study, and some were preselected as having demonstrable BAT. Older, obese humans often have little or no demonstrable BAT $(16,52)$. One study did demonstrate detectable BAT in obese men but used a prolonged cold exposure protocol (53). In this study, the inability of mirabegron treatment to recruit BAT is probably because of the low levels of BAT at baseline in this older, obese cohort.

Although adults with obesity have little BAT, there is considerable WAT, and both cold and mirabegron stimulate SC WAT beiging, with increases in UCP1, CIDEA, and TMEM26 (30). In spite of a clear mirabegron-induced increase in UCP1 protein expression in SC WAT, we did not detect an increase in uncoupled respiration in purified mitochondria from WAT. In a previous study using the same methods, cold exposure of lean subjects increased uncoupled respiration in mitochondria isolated from SC WAT with a similar level of induction of UCP1 (30). The reasons for the failure of mirabegron to induce uncoupling activity are unclear, but it could be that mirabegron induced CIDEA, an inhibitor of UCP1 activity (54). Mirabegron may have affected ROS levels, which are necessary to activate UCP1 (55).

Although there was no increase in uncoupled respiration with mirabegron, the increase in lipolysis observed in this study and the previously reported increase in HSL phosphorylation on a serine residue that is a protein kinase A site (serine 660) (30) suggest that mirabegron stimulated cAMP signaling in SC WAT. Furthermore, mirabegron treatment caused numerous changes in the expression of genes, most of which are predicted to reduce adipose tissue dysfunction. To gain a mechanistic understanding of the improvement in glucose metabolism, we attempted to identify changes in plasma levels of adipokines and in proteins whose adipose gene expression was altered by mirabegron. For instance, FGF21 has many favorable metabolic effects and was upregulated in SC WAT after mirabegron treatment (56). Resistin was initially discovered 
A

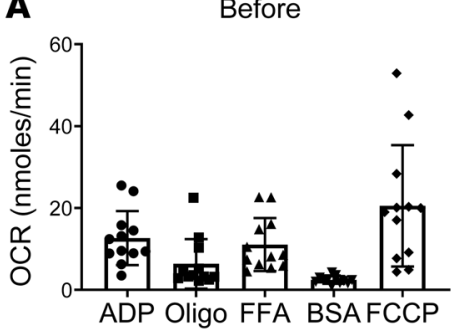

B

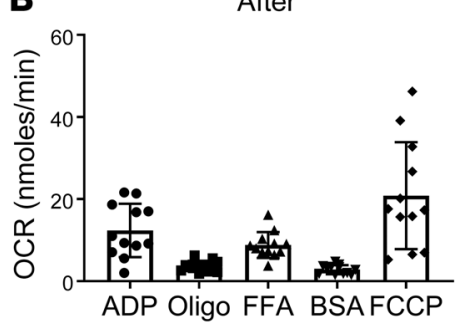

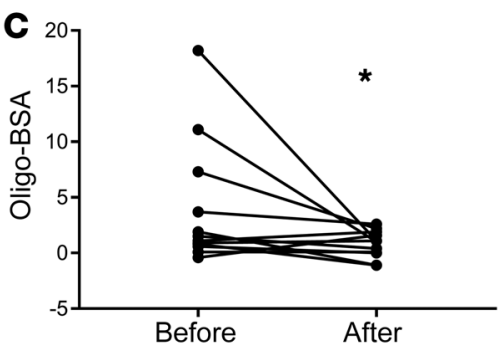

Figure 7. Mirabegron treatment reduces state 4 respiration but does not increase uncoupled respiration in purified mitochondria isolated from SC WAT. Mitochondria were purified and the bioenergetics analyzed using an Oxytherm before and after mirabegron treatment as described in Methods. This involved the sequential addition of adenosine diphosphate (ADP), oligomycin (Oligo), FFA (60 $\mu$ M linoleic acid), fatty acid-free BSA, and trifluoromethoxy carbonylcyanide phenylhydrazone (FCCP) $(10 \mu \mathrm{m})$. (A and B) OCRs before and after treatment are shown. (C) The difference between oligomycin and BSA was calculated before and after treatment. Data indicate the mean $\pm \operatorname{SEM}(n=12) .{ }^{*} P=0.05$, by Wilcoxon matched-pairs, signed-rank test.

to be a factor secreted by adipocytes that causes insulin resistance in mice (57). Studies of resistin in humans initially yielded conflicting results; however, 2 large studies identified resistin as a risk factor for the development of diabetes $(58,59)$. We were not able to identify changes in the plasma levels of FGF21, resistin, adiponectin, leptin, or adipsin. Overall, our findings suggest that mirabegron treatment affected many different pathways that have an impact on adipose tissue function, and it will be important to identify mechanisms linking changes in SC WAT to improved glucose homeostasis in future studies.

One of the most interesting findings from this study was the change in skeletal muscle fiber types. The switch in muscle fiber type induced by mirabegron treatment is rather remarkable, since muscle fiber type is relatively resistant to a transition from type II to type I, even with significant interventions such as exercise and weight loss $(60,61)$. The $\beta 2-A R$ is expressed in muscle, and administration of $\beta 2$-AR agonists causes hypertrophy as well as a shift from type I to type II fibers $(62,63)$. We did not detect an increase in fiber size or type I to type II switching, indicating that mirabegron did not activate $\beta 2$-ARs, consistent with the lack of cardiovascular side effects. Mirabegron increased PGC1A mRNA levels as well as the expression of genes regulated by PGC1 $\alpha$ PGC1 $\alpha$ promotes type I fiber induction in mice (38), and the increase in PGC1A after mirabegron treatment is thus likely important for a number of changes including observations that lipotoxicity was not increased despite increased plasma NEFAs and that insulin sensitivity was increased. It will therefore be important to identify the mechanism for PGC1A induction by mirabegron treatment and to consider that beige adipose may be the source of the factor.

$\beta$ Cell function was increased by mirabegron treatment. A recent study in mice demonstrated an acute effect of $\beta 3$-AR agonism on insulin secretion, and this effect was entirely dependent on adipose tissue lipolysis, since it was completely blocked in mice with adipose tissue triglyceride lipase (ATGL) deficiency (45). This observation suggests that the $\beta 3$-AR is not expressed on pancreatic $\beta$ cells in rodents and illustrates a communication between adipose tissue and $\beta$ cells. In addition to NEFAs, adiponectin, leptin, and adipsin are proteins secreted from adipose tissue that have an impact on $\beta$ cells (64-66). The results of our study indicate that the change in the disposition index, a measure of $\beta$ cell function, correlated with both the change in SC WAT UCP1 expression and the change in plasma NEFA levels, suggesting a relationship between the effect of mirabegron on SC WAT and $\beta$ cell function. It is possible that this effect is mediated directly by increased NEFAs. It will be important to determine whether this effect persists under longer-term mirabegron treatment. Although NEFAs stimulate $\beta$ cell insulin secretion acutely, the compensatory mechanisms of $\beta$ cells during chronic exposure to increased NEFAs are detrimental to insulin secretion (41-43). Another possibility is that stimulation of beige adipose results in the release of additional factors that protect $\beta$ cell function.

A leading hypothesis for the development of adipose dysfunction in obesity is adipocyte expansion against a rigid ECM, leading to adipocyte necrosis and macrophage recruitment $(24,25,67)$. Mirabegron treatment resulted in reduced mRNA expression of collagen VI and several genes involved in adipose tissue remodeling. Although mirabegron treatment induced lipolysis, we did not observe any change in adipocyte size (data not shown). This suggests that the changes in ECM gene expression may be due to the induction of beiging, which is linked to reduced fibrosis and a less rigid ECM $(11,23)$. In addition to the changes in ECM genes, the number of alternatively activated macrophages $\left(\mathrm{CD} 163^{+} \mathrm{CD} 68^{+}\right)$ increased in SC WAT after mirabegron treatment, suggesting an environment that is less inflammatory and more reparative. Macrophages have been reported to be recruited into adipose tissue in response to increased lipolysis to buffer FFAs (68). We observed that the $\mathrm{CD} 163^{+} \mathrm{CD} 68^{+}$macrophages recruited into SC WAT expressed UCP1. Although uncoupling activity in the adipose tissue did not increase overall, it is possible that UCP1 was active in these macrophages as a mechanism of oxidizing lipids. UCP1 was also expressed in $\mathrm{CD}_{206^{+}}$macrophages, which increased after mirabegron treatment but did not reach statistical significance (data not shown; $P=0.12$ ). The identification of UCP1 in adipose tissue macrophages (Figure 6C) partly explains the punctate nature of UCP1 staining that we observed in human WAT.

Limitations of the study. This study was designed to be a proof of concept, since, to our knowledge, $\beta 3$-AR agonists have not been studied in obese humans, and we sought to comprehensively characterize the response to mirabegron using both basic science and clinical techniques. Furthermore, this study was not placebo controlled, and the majority of the subjects were female. A randomized, blinded, placebo-controlled study with a larger number 
of participants and males will be necessary to determine whether there are sex differences and to identify small changes that may have been missed in this study. For instance, our data indicate that mirabegron at the FDA-approved dose of $50 \mathrm{mg}$ /day is specific for $\beta 3$-ARs, which is based on a lack of cardiovascular side effects. With the sample size of this study, we cannot completely rule out $\beta 2$-AR agonism, which could, for instance, affect $\beta$ cell function directly or indirectly via other cells in the islet. A placebo control group will also be necessary to rule out a placebo effect for some of the small changes identified in this study, such as the small but consistent increase in GIR. Despite these limitations, we believe this study forms a strong basis for future studies on mirabegron, as described below.

Conclusions, perspectives, and future goals. $\beta 3$-AR agonists were initially developed as antiobesity agents. Although $\beta 3$-AR agonists induce weight loss in rodents, we did not observe weight loss in the subjects in this study. This could be due to the relatively low levels of BAT in humans, the mirabegron dose used, or the fact that humans will compensate for increased energy expenditure with increased food intake. Alternatively, it is possible that the $50-\mathrm{mg} /$ day dose was too low to induce beiging and UCP1 activation to a level that would increase energy expenditure and weight loss. However, even without weight loss or BAT activation, this relatively low dose was sufficient to improve insulin sensitivity and $\beta$ cell function, despite the fact that NEFA levels increased. Higher doses of mirabegron would likely induce stronger beiging and increased lipid oxidation, which may reduce NEFAs; however, unwanted cardiovascular side effects may be limiting $(31,32)$. It will be important to determine whether long-term mirabegron treatment at the dose used in this study would maintain improved $\beta$ cell function and delay the onset of diabetes in prediabetic subjects. Finally, it would be important to know whether mirabegron improves glucose homeostasis in individuals with type 2 diabetes.

\section{Methods}

Study design and human subjects. This study was intended to examine the metabolic effects of mirabegron in obese, insulin-resistant humans at the dose approved by the FDA for overactive bladder. Hence, we recruited 13 sedentary subjects, aged 35 to 65 years, who had a BMI over 27 and either prediabetes, on the basis of HbA1c and a standard 75 g OGTT, or normal glucose tolerance (NGT) but more than 3 features of metabolic syndrome (MetS). Nine of the 13 subjects met at least 1 criterion for prediabetes at baseline: impaired fasting glucose (100-125 $\mathrm{mg} / \mathrm{dL}$ ), impaired glucose tolerance (120-minute glucose: 140-199 $\mathrm{mg} / \mathrm{dL}$ ), or HbA1c of $5.7 \%$ to $6.4 \%$. In addition to the OGTT, baseline studies were performed in fasted subjects early in the morning in a quite, temperature-controlled room $\left(22^{\circ} \mathrm{C}\right)$ and included measurement of body composition by dual-energy x-ray absorptiometry (DEXA) scan and resting metabolic rate (RMR) with a metabolic cart. Other baseline studies included a euglycemic clamp, SC WAT and muscle (vastus lateralis) biopsies, and PET-CT scans under cold stimulation to quantify BAT.

The subjects were recruited from the Lexington, Kentucky, area throughout the year, and all subjects had normal thyroid function, no chronic inflammatory conditions, and were not taking any medications, such as beta blockers, steroids, or insulin sensitizers, that would interfere with the study. The subjects were asked to continue their usual diet and level of activity. After completing the baseline studies (OGTT, euglycemic clamp, fat and muscle biopsies, and PET-CT), the participants were treated with mirabegron $(50 \mathrm{mg} /$ day) for 12 weeks, after which the initial studies were repeated. The Clinicaltrials.gov identifier is NCT02919176.

Biopsies. An incisional biopsy of adipose tissue from the abdomen was obtained as described previously (69). Briefly, approximately 4 grams of tissue was removed from the abdomen, most of which was used for the preparation of mitochondria for mitochondrial bioenergetics analysis (30). The remainder was either frozen at $-80^{\circ} \mathrm{C}$ or used for immunohistochemical analyses. A muscle biopsy from the vastus lateralis was also obtained using a Bergstrom needle as described previously (70). Briefly, approximately $100 \mathrm{mg}$ of each biopsy was mounted with fibers perpendicular to a cork using tragacanth gum; this was snap-frozen in liquid nitrogen-cooled isopentane and then stored at $-80^{\circ} \mathrm{C}$ until sectioning.

Euglycemic clamp. Peripheral insulin sensitivity was measured using a euglycemic clamp, as described previously (71). The subjects arrived in the fasted state, IVs were inserted, and euglycemia was maintained through the variable infusion of $20 \%$ glucose and frequent blood glucose measurement during an insulin infusion of $1.0 \mathrm{mU} / \mathrm{kg} /$ min. A steady state was generally attained at 2 hours, and the glucose infusion rate was determined during the final 30 minutes.

Assessment of BAT. Cold-stimulated PET-CT for the measurement of BAT was performed as described previously $(31,72)$. After an overnight fast, the subjects were brought to the PET-CT suite 120 minutes before the scan and outfitted with a cooling vest that circulated water at $14^{\circ} \mathrm{C}$. Sixty minutes before the scan, $440 \mathrm{MBq}{ }^{18} \mathrm{~F}-\mathrm{FDG}$ was injected intravenously. Images were acquired using a Discovery LS Multidetector Helical PET-CT Scanner (GE Medical Systems). PET data were acquired from the eyes to the thighs, and both PET and CT images were reconstructed as axial sections at $4.25-\mathrm{mm}$ spacing and then reformatted by the viewer software. BAT mass and activity were quantified using the PET-CT Viewer shareware, and BAT in each axial slice was classified on a pixel-by-pixel basis when CT was in the range of -250 to -10 Hounsfield units and when the maximal standard uptake value (SUVmax) was 2.0 or higher in the cervical, supraclavicular, and anterior thoracic depots from C3 to T7. Areas of ${ }^{18} \mathrm{~F}-\mathrm{FDG}$ uptake on PET colocalizing with regions of fat identified on CT were quantified by their average SUV (SUVavg), and detectable BAT volume was calculated as the sum of pixels meeting this classification criteria multiplied by the pixel volume $\left(64.8 \mathrm{~mm}^{3}\right)$.

IHC on adipose tissue and muscle. IHC and quantification of UCP1, TMEM26, and CIDEA expression in SC WAT were performed as described previously (30). We performed immunohistochemical analyses of macrophages $\left(\mathrm{CD} 163^{+} \mathrm{CD} 68^{+}, \mathrm{CD}^{2} 06^{+} \mathrm{CD}^{+} 8^{+}\right.$, or $\mathrm{CD}^{2} 6^{+} \mathrm{CD} 68^{+}$) on $5-\mu \mathrm{m}$ formalin-fixed, paraffin-embedded SC WAT sections. Tissue was deparaffinized and blocked with hydrogen peroxide, rinsed, and then blocked first with a Streptavidin/Biotin Blocking Kit (SP-2002, Vector Laboratories) followed by $2.5 \%$ horse serum. M1 macrophages were identified by costaining with rabbit anti-CD86 (ab53004, Abcam) and mouse anti-CD68 (ab955, Abcam) antibodies; M2 macrophages were identified by costaining with rabbit antiCD163 (ab182422, Abcam) and anti-CD68 antibodies. The primary antibodies were incubated individually overnight in $1 \%$ horse serum. The number of CD163 macrophages that costained with UCP1 was determined using mouse anti-CD163 (HM2157, Hycult Biotech) and 
rabbit anti-UCP1 (custom antibody J2648, ECM Biosciences). Amplification was performed with either goat anti-mouse IgG biotin (115065-205, Jackson ImmunoResearch) or donkey anti-rabbit IgG biotin (711-065-152, Jackson ImmunoResearch) and then streptavidin-HRP (S911, Thermo Fisher Scientific), followed by washing and incubation with Alexa Fluor Tyramide Reagent (Thermo Fisher Scientific). Sections were mounted and nuclei stained using VECTASHIELD Antifade Mounting Media with DAPI (H-1800, Vector Laboratories). Macrophages were counted when cells were triple-stained with DAPI and CD68, CD86, or CD163. UCP1 ${ }^{+} \mathrm{CD} 163^{+}$cells were triple-stained with CD163, UCP1, and DAPI. Macrophages were counted within fields of adipocytes and not in connective tissue. All data were normalized to adipocyte numbers. Images were captured with an upright Zeiss fluorescence microscope.

We performed immunohistochemical analyses on muscle biopsies obtained from the vastus lateralis as described previously (73). Briefly, frozen muscle sections were cut at $8 \mu \mathrm{m}$, air-dried, and stored at $-20^{\circ} \mathrm{C}$. Sections were incubated overnight with isotype-specific anti-mouse antibodies against MyHC I IgG2B (BA.D5), MyHC IIa IgG1 (SC.71), and MyHC IIx (6H1), obtained from the Developmental Studies Hybridoma Bank. Sections were then incubated with the following secondary antibodies: goat anti-mouse IgG2b Alexa Fluor 647, anti-mouse IgG1 Alexa Fluor 488, and anti-mouse IgM Alexa Fluor 555 (Invitrogen, Thermo Fisher Scientific). The sections were then mounted using VECTASHIELD Anti-fade Mounting Media and postfixed in methanol. Five to 7 images were captured at $\times 20$ magnification using an AxioImager M1 upright fluorescence microscope (Zeiss). Fiber type distribution was quantified manually in a blinded manner.

mRNA quantification. Muscle and adipose mRNA was isolated using TRIzol extraction and purification with RNeasy Lipid Tissue Mini Kits (QIAGEN). The quantity and quality were RNA determined using an Agilent 2100 Bioanalyzer. Multiplex analysis of SC WAT gene expression was performed using the NanoString nCounter system (Nanostring) and a custom code set. The data were normalized to the geometric mean of 6 housekeeping genes; the genes and accession numbers of the NanoString custom code set are provided in Supplemental Table 5. Muscle gene expression was analyzed by real-time RT-PCR as described previously (69), and the data were normalized to the geometric mean of 6 housekeeping genes ( $A C T B, P P I A, P P I B$, $T B P, T U B B$, and $U B C 9)$. The primer sequences are shown in Supplemental Table 6.

Quantification of lipids in muscle biopsies. Approximately 10 milligrams of the vastus lateralis biopsy was weighed and then extracted with acidified organic solvents. DAGs and ceramides were quantified as described perviously (74). TGs were quantified in the extract using colorimetric TG assays (T7532, Point Scientific). Briefly, lipid extract $(10 \mu \mathrm{L})$ was dried for 5 minutes, and $10 \mu \mathrm{L} 0.1 \%$ SDS in $0.9 \% \mathrm{NaCl}$ was added and then incubated for 10 minutes at $37^{\circ} \mathrm{C}$. Prewarmed TG reagent $(1 \mathrm{~mL})$ was added, and the reaction was incubated at $37^{\circ} \mathrm{C}$ for 5 minutes. The absorbance $\left(\mathrm{A}_{500}\right)$ was measured with a Bio-Rad spectrophotometer within 30 minutes. Lipid levels were normalized to the tissue weight.

Myotube treatment with adipocyte CM. Adult-derived human adipocyte stem cells (ADHASCs) were cultured and differentiated as described previously (75). In brief, preadipocytes were incubated for 48 hours after reaching confluence and induced to differentiate into adipocytes with adipocyte differentiation medium (50\% DMEM:
50\% Ham's F-10); 3\% FBS (Invitrogen, Thermo Fisher Scientific); $15 \mathrm{mM}$ HEPES, pH 7.4, $33 \mu \mathrm{M}$ biotin (MilliporeSigma); $17 \mu \mathrm{M}$ pantothenate (MilliporeSigma); $1 \mu \mathrm{M}$ dexamethasone (MilliporeSigma); 0.25 mM 3-isobutyl-1-methylxanthine (IBMX) (MilliporeSigma); $1 \times$ $10^{-7} \mathrm{M}$ insulin (Novo Nordisk); and $1 \mu \mathrm{M}$ rosiglitazone (SmithKline Beecham) for 3 days. The cells were then incubated with adipocyte differentiation medium without IBMX and rosiglitazone, with medium changes every 3 days until $80 \%-90 \%$ of the cells had lipid droplets (7-10 days). Adipocyte CM was prepared by incubating differentiated ADHASCs with DMEM with 2\% FBS for 24 hours and then with DMEM with 2\%FBS and 0.1\% DMSO (vehicle control) or $100 \mathrm{nM}$ mirabegron (Cayman Chemical) for 16 hours. CM was also isolated and mirabegron added to this medium (100 nM). Adipocyte $\mathrm{CM}$ was filtered with a $0.2-\mu \mathrm{m}$ filter and frozen at $-80^{\circ} \mathrm{C}$ for later use. Primary myoblasts from human skeletal muscle biopsies from healthy subjects were provided by Charlotte Peterson (University of Kentucky, Lexington, Kentucky, USA). The myoblasts were differentiated in DMEM with 2\% FBS for 10 days. Fully differentiated myotubes were treated with $25 \mathrm{nM}$ mirabegron or $25 \%$ adipocyte $\mathrm{CM}$ for 16 hours, and total RNA was prepared for mRNA expression analysis using real-time RT-PCR.

NEFA and glycerol measurements in plasma and adipose explant secretions. Adipose explant secretions were prepared by incubating 0.5 grams of the SC WAT biopsy in $2 \mathrm{~mL}$ Ringer's solution supplemented with $1 \%$ lipid-free BSA at $37^{\circ} \mathrm{C}$ for 1 hour. Glycerol in the explant secretion was then measured with a High-Sensitivity Free Glycerol Fluorometric Assay Kit (MAK270, MilliporeSigma). Glycerol in plasma was measured with Free Glycerol Reagent (F6428, MilliporeSigma). NEFAs in plasma or adipose explant secretions were measured with HR Series NEFA-HR(2) Color Reagents (999-34691, 995-34791, 99134891, 993-35191, FUJIFILM Wako Diagnostics)

Mitochondrial bioenergetics. Bioenergetics of mitochondria purified from the SC WAT biopsies were determined using an Oxytherm System (Hansatech) as previously described (30).

Statistics. A paired Student's $t$ test or Wilcoxon matched-pairs, signed-rank test was performed to compare the results before and after mirabegron treatment. Oral glucose tolerance was assessed by a repeated-measures 2-way ANOVA with Sidak's multiple comparisons test. Spearman's correlations were used to assess associations between beiging, plasma NEFA levels, and $\beta$ cell function. Multiple comparisons were analyzed by 1-way ANOVA and Tukey's multiple comparisons test. Data were analyzed using GraphPad Prism, version 8.0 (GraphPad Software). All tests were 2 sided, and statistical significance was set at a $P$ value of 0.05 or less.

Study approval. All subjects provided informed consent, and the protocols were approved by the IRB of the University of Kentucky.

\section{Author contributions}

PAK, EEDV, PGS, AJM, and BSF designed the experiments, analyzed data, and wrote the manuscript. BZ, HM, ALC, ZRJ, JC, and HJV performed experiments. PMW analyzed data. RHEK performed cold-stimulated PET CT studies and analyzed data.

\section{Acknowledgments}

We wish to thank the staff of the University of Kentucky Clinical Research Unit for assistance with this study and Dorothy Ross for coordinating the recruitment of the participants. We acknowl- 
edge Charlotte Peterson for providing the primary myoblasts and Rachel Davidowitz for her help with the graphical abstract. This study was funded by NIH grants DK112282, P30GM127211, and DK 71349 and by CTSA, NIH grant UL1TR001998.
Address correspondence to: Philip A. Kern, Division of Endocrinology, CTW 521, University of Kentucky, 900 South Limestone Street, Lexington, Kentucky 40536, USA. Phone: 859.218.1394; philipkern@uky.edu.
1. Arch JR. Challenges in $\beta$ (3)-adrenoceptor agonist drug development. Ther Adv Endocrinol Metab. 2011;2(2):59-64.

2. Arch JR. beta(3)-Adrenoceptor agonists: potential, pitfalls and progress. Eur J Pharmacol. 2002;440(2-3):99-107.

3. Arch JR, et al. Treatment of obesity with thermogenic beta-adrenoceptor agonists: studies on BRL 26830A in rodents. Int JObes. 1984;8 Suppl 1:1-11.

4. Arch JR, Ainsworth AT. Thermogenic and antiobesity activity of a novel beta-adrenoceptor agonist (BRL 26830A) in mice and rats. Am JClin Nutr. 1983;38(4):549-558.

5. Hao L, et al. Beneficial metabolic effects of mirabegron in vitro and in high-fat diet-induced obese mice. JPharmacol Exp Ther. 2019;369(3):419-427.

6. Frontini A, Cinti S. Distribution and development of brown adipocytes in the murine and human adipose organ. Cell Metab. 2010;11(4):253-256.

7. Wu J, et al. Beige adipocytes are a distinct type of thermogenic fat cell in mouse and human. Cell. 2012;150(2):366-376.

8. Seale P, et al. Prdm16 determines the thermogenic program of subcutaneous white adipose tissue in mice. J Clin Invest. 2011;121(1):96-105.

9. Cohen P, et al. Ablation of PRDM16 and beige adipose causes metabolic dysfunction and a subcutaneous to visceral fat switch. Cell. 2014;156(1-2):304-316.

10. Min SY, et al. Human 'brite/beige' adipocytes develop from capillary networks, and their implantation improves metabolic homeostasis in mice. Nat Med. 2016;22(3):312-318.

11. Hasegawa $Y$, et al. Repression of adipose tissue fibrosis through a PRDM16-GTF2IRD1 complex improves systemic glucose homeostasis. Cell Metab. 2018;27(1):180-194.e6.

12. Chen Y, et al. Thermal stress induces glycolytic beige fat formation via a myogenic state. Nature. 2019;565(7738):180-185.

13. Cannon B, Nedergaard J. Brown adipose tissue: function and physiological significance. Physiol Rev. 2004;84(1):277-359.

14. van Marken Lichtenbelt WD, et al. Cold-activated brown adipose tissue in healthy men. $N$ EnglJ Med. 2009;360(15):1500-1508.

15. Virtanen KA, et al. Functional brown adipose tissue in healthy adults. N EnglJMed. 2009;360(15):1518-1525.

16. Saito M, et al. High incidence of metabolically active brown adipose tissue in healthy adult humans: effects of cold exposure and adiposity. Diabetes. 2009;58(7):1526-1531.

17. Cypess AM, et al. Identification and importance of brown adipose tissue in adult humans. $\mathrm{N}$ EnglJ Med. 2009;360(15):1509-1517.

18. Klepac K, Georgiadi A, Tschöp M, Herzig S. The role of brown and beige adipose tissue in glycaemic control. Mol Aspects Med. 2019;68:90-100.

19. Porter C, Chondronikola M, Sidossis LS. The therapeutic potential of brown adipocytes in humans. Front Endocrinol (Lausanne). 2015;6:156

20. Kajimura S, Spiegelman BM, Seale P. Brown and beige fat: physiological roles beyond Heat Generation. Cell Metab. 2015;22(4):546-559.

21. Ikeda K, et al. UCP1-independent signaling involving SERCA2b-mediated calcium cycling regulates beige fat thermogenesis and systemic glucose homeostasis. Nat Med. 2017;23(12):1454-1465.

22. Kazak L, et al. A creatine-driven substrate cycle enhances energy expenditure and thermogenesis in beige fat. Cell. 2015;163(3):643-655.

23. Wang $\mathrm{W}$, et al. A PRDM16-driven metabolic signal from adipocytes regulates precursor cell fate. Cell Metab. 2019;30(1):174-189.e5.

24. Crewe C, An YA, Scherer PE. The ominous triad of adipose tissue dysfunction: inflammation, fibrosis, and impaired angiogenesis. J Clin Invest. 2017;127(1):74-82.

25. Sun K, Kusminski CM, Scherer PE. Adipose tissue remodeling and obesity. J Clin Invest. 2011;121(6):2094-2101.

26. Sidossis LS, et al. Browning of subcutaneous white adipose tissue in humans after severe adrenergic stress. Cell Metab. 2015;22(2):219-227.

27. Petruzzelli M, et al. A switch from white to brown fat increases energy expenditure in cancer-associated cachexia. Cell Metab. 2014;20(3):433-447.

28. Frontini A, et al. White-to-brown transdifferentiation of omental adipocytes in patients affected by pheochromocytoma. Biochim Biophys Acta. 2013;1831(5):950-959.

29. Kir S, et al. Tumour-derived PTH-related protein triggers adipose tissue browning and cancer cachexia. Nature. 2014;513(7516):100-104.

30. Finlin BS, et al. Human adipose beiging in response to cold and mirabegron. JCI Insight. 2018;3(15):121510.

31. Cypess AM, et al. Activation of human brown adipose tissue by a $\beta 3$-adrenergic receptor agonist. Cell Metab. 2015;21(1):33-38.

32. Baskin AS, et al. Regulation of human adipose tissue activation, gallbladder size, and bile acid metabolism by a $\beta 3$-adrenergic receptor agonist. Diabetes. 2018;67(10):2113-2125.

33. Loh RKC, Formosa MF, La Gerche A, Reutens AT, Kingwell BA, Carey AL. Acute metabolic and cardiovascular effects of mirabegron in healthy individuals. Diabetes Obes Metab. 2019;21(2):276-284.

34. Nitti VW, et al. Safety and tolerability of the $\beta 3$-adrenoceptor agonist mirabegron, for the treatment of overactive bladder: results of a prospective pooled analysis of three 12 -week randomised phase III trials and of a 1-year randomised phase III trial. Int J Clin Pract. 2014;68(8):972-985.

35. Pisprasert V, Ingram KH, Lopez-Davila MF, Munoz AJ, Garvey WT. Limitations in the use of indices using glucose and insulin levels to predict insulin sensitivity: impact of race and gender and superiority of the indices derived from oral glucose tolerance test in African Americans. Diabetes Care. 2013;36(4):845-853.

36. Bergman RN, Finegood DT, Kahn SE. The evolution of beta-cell dysfunction and insulin resistance in type 2 diabetes. Eur J Clin Invest. 2002;32 Suppl 3:35-45.

37. Fan W, Atkins AR, Yu RT, Downes M, Evans RM. Road to exercise mimetics: targeting nuclear receptors in skeletal muscle. JMol Endocrinol. 2013;51(3):T87-T100.

38. Lin J, et al. Transcriptional co-activator PGC-1 alpha drives the formation of slow-twitch muscle fibres. Nature. 2002;418(6899):797-801.

39. Mason RR, Watt MJ. Unraveling the roles of PLIN5: linking cell biology to physiology. Trends Endocrinol Metab. 2015;26(3):144-152.

40. Hinkle RT, Hodge KM, Cody DB, Sheldon RJ, Kobilka BK, Isfort RJ. Skeletal muscle hypertrophy and anti-atrophy effects of clenbuterol are mediated by the beta2-adrenergic receptor. $\mathrm{Mus}$ cle Nerve. 2002;25(5):729-734.

41. Nolan CJ, Madiraju MS, Delghingaro-Augusto V, Peyot ML, Prentki M. Fatty acid signaling in the beta-cell and insulin secretion. Diabetes. 2006;55 Suppl 2:S16-S23.

42. Prentki M, Matschinsky FM, Madiraju SR. Metabolic signaling in fuel-induced insulin secretion. Cell Metab. 2013;18(2):162-185.

43. Ježek P, Jabůrek M, Holendová B, Plecitá-Hlavatá L. Fatty acid-stimulated insulin secretion vs. lipotoxicity. Molecules. 2018;23(6):E1483.

44. Distel RJ, Robinson GS, Spiegelman BM. Fatty acid regulation of gene expression. Transcriptional and post-transcriptional mechanisms. J Biol Chem. 1992;267(9):5937-5941.

45. Heine $\mathrm{M}$, et al. Lipolysis triggers a systemic insulin response essential for efficient energy replenishment of activated brown adipose tissue in mice. Cell Metab. 2018;28(4):644-655.e4.

46. Magkos F, et al. Effects of moderate and subsequent progressive weight loss on metabolic function and adipose tissue biology in humans with obesity. Cell Metab. 2016;23(4):591-601.

47. Sui $\mathrm{W}$, et al. Bladder drug mirabegron exacerbates atherosclerosis through activation of brown fat-mediated lipolysis. Proc Natl Acad Sci USA. 2019;116(22):10937-10942.

48. Hanssen MJ, et al. Short-term cold acclimation improves insulin sensitivity in patients with type 2 diabetes mellitus. Nat Med. 2015;21(8):863-865.

49. van der Lans AA, et al. Cold acclimation recruits human brown fat and increases nonshivering thermogenesis. J Clin Invest. 2013;123(8):3395-3403.

50. Yoneshiro T, et al. Recruited brown adipose tissue as an antiobesity agent in humans. J Clin Invest. 2013;123(8):3404-3408.

51. Hanssen MJ, et al. Short-term cold acclimation recruits brown adipose tissue in obese humans. Diabetes. 2016;65(5):1179-1189. 
52. Vijgen GH, Bouvy ND, Teule GJ, Brans B, Schrauwen P, van Marken Lichtenbelt WD. Brown adipose tissue in morbidly obese subjects. PLOS ONE. 2011;6(2):e17247.

53. Chondronikola $\mathrm{M}$, et al. Brown adipose tissue activation is linked to distinct systemic effects on lipid metabolism in humans. Cell Metab. 2016;23(6):1200-1206.

54. Fischer AW, et al. UCP1 inhibition in Cidea-overexpressing mice is physiologically counteracted by brown adipose tissue hyperrecruitment. Am J Physiol Endocrinol Metab. 2017;312(1):E72-E87.

55. Chouchani ET, et al. Mitochondrial ROS regulate thermogenic energy expenditure and sulfenylation of UCP1. Nature. 2016;532(7597):112-116.

56. Fisher FM, Maratos-Flier E. Understanding the physiology of FGF21. Annu Rev Physiol. 2016;78:223-241.

57. Schwartz DR, Lazar MA. Human resistin: found in translation from mouse to man. Trends Endocrinol Metab. 2011;22(7):259-265.

58. Chen BH, et al. Circulating levels of resistin and risk of type 2 diabetes in men and women: results from two prospective cohorts. Diabetes Care. 2009;32(2):329-334.

59. Heidemann C, et al. Total and high-molecular-weight adiponectin and resistin in relation to the risk for type 2 diabetes in women. Ann Intern Med. 2008;149(5):307-316.
60. Kern PA, Simsolo RB, Fournier M. Effect of weight loss on muscle fiber type, fiber size, capillarity, and succinate dehydrogenase activity in humans. JClin Endocrinol Metab. 1999;84(11):4185-4190.

61. Wilson JM, Loenneke JP, Jo E, Wilson GJ, Zourdos MC, Kim JS. The effects of endurance, strength, and power training on muscle fiber type shifting. J Strength Cond Res. 2012;26(6):1724-1729.

62. Ryall JG, Lynch GS. The potential and the pitfalls of beta-adrenoceptor agonists for the management of skeletal muscle wasting. Pharmacol Ther. 2008;120(3):219-232.

63. Ohnuki Y, et al. Role of masseter muscle $\beta_{2}$-adrenergic signaling in regulation of muscle activity, myosin heavy chain transition, and hypertrophy. J Pharmacol Sci. 2013;123(1):36-46.

64. Lo JC, et al. Adipsin is an adipokine that improves $\beta$ cell function in diabetes. Cell. 2014;158(1):41-53.

65. Stern JH, Rutkowski JM, Scherer PE. Adiponectin, leptin, and fatty acids in the maintenance of metabolic homeostasis through adipose tissue crosstalk. Cell Metab. 2016;23(5):770-784.

66. Holland WL, et al. Receptor-mediated activation of ceramidase activity initiates the pleiotropic actions of adiponectin. Nat Med. 2011;17(1):55-63.

67. Sun K, Tordjman J, Clément K, Scherer PE. Fibrosis and adipose tissue dysfunction. Cell Metab. 2013;18(4):470-477.
68. Kosteli A, et al. Weight loss and lipolysis promote a dynamic immune response in murine adipose tissue. J Clin Invest. 2010;120(10):3466-3479.

69. Finlin BS, et al. Mast cells promote seasonal white adipose beiging in humans. Diabetes. 2017;66(5):1237-1246.

70. Walton RG, et al. Human skeletal muscle macrophages increase following cycle training and are associated with adaptations that may facilitate growth. Sci Rep. 2019;9(1):969.

71. Finlin BS, et al. Effect of rifaximin treatment on endotoxemia and insulin sensitivity in humans. JEndocr Soc. 2019;3(9):1641-1651.

72. Chen KY, et al. Brown adipose reporting criteria in imaging studies (BARCIST 1.0): recommendations for standardized FDG-PET/CT experiments in humans. Cell Metab. 2016;24(2):210-222.

73. Murach KA, et al. Depletion of $\operatorname{Pax} 7^{+}$satellite cells does not affect diaphragm adaptations to running in young or aged mice. J Physiol. 2017;595(19):6299-6311.

74. Walton RG, et al. Increasing adipocyte lipoprotein lipase improves glucose metabolism in high fat diet-induced obesity. J Biol Chem. 2015;290(18):11547-11556.

75. Rasouli N, et al. Association of scavenger receptors in adipose tissue with insulin resistance in nondiabetic humans. Arterioscler Thromb Vasc Biol. 2009;29(9):1328-1335. 